\title{
Computed tomography-guided percutaneous biopsy for vertebral neoplasms: a department's experience and hybrid biopsy technique to improve yield
}

\author{
Vasant Garg, MD, ${ }^{1}$ Christos Kosmas, MD, ${ }^{1}$ Enambir S. Josan, MBBS, ${ }^{2}$ Sasan Partovi, MD, \\ Nicholas Bhojwani, MD, ${ }^{1}$ Nathan Fergus, MD, ${ }^{1}$ Peter C. Young, MD, ${ }^{1}$ and Mark R. Robbin, MD ${ }^{1}$ \\ 1'Department of Radiology, University Hospitals Case Medical Center, and ${ }^{2}$ Case Western Reserve University School of \\ Medicine, Cleveland, Ohio
}

OBJECTIVE Recent articles have identified the poor diagnostic yield of percutaneous needle biopsy for vertebral osteomyelitis. The current study aimed to confirm the higher accuracy of CT-guided spinal biopsy for vertebral neoplasms and to identify which biopsy technique provides the highest yield.

METHODS Over a 9-year period, the radiology department at University Hospitals Case Medical Center performed 222 CT-guided biopsies of vertebral lesions, of which clinicians indicated a concern for vertebral neoplasms in 122 patients. A retrospective chart review was performed to confirm the higher sensitivity of the percutaneous intervention for vertebral neoplasms.

RESULTS A core sample was obtained for all 122 biopsies of concern (100.0\%). Only 6 cases $(4.9 \%)$ were reported as nondiagnostic per histological sampling, and 12 cases $(9.8 \%)$ were negative for disease. The question of vertebral neoplastic involvement warrants follow-up, and the current study was able to determine the subsequent diagnosis of each lesion. Of the 122 total, 94 (77.0\%) core samples provided true-positive results, and the sensitivity of core biopsy measured $87.9 \%$. The technical approach did not demonstrate any significant difference in diagnostic yield. However, when the vertebral cortex was initially pierced with a coaxial bone biopsy system and subsequently a 14-gauge spring-loaded cutting biopsy needle was coaxially advanced into lytic lesions, 14 true positives were obtained with a corresponding sensitivity of $100.0 \%$.

CONCLUSIONS This study confirms the higher sensitivity of image-guided percutaneous needle biopsy for vertebral neoplasms. In addition, it demonstrates how the use of a novel cutting needle biopsy approach, performed coaxially through a core biopsy track, provides the highest yield.

http://thejns.org/doi/abs/10.3171/2016.4.FOCUS1614

KEY WORDS CT-guided biopsy; vertebral neoplasms; hybrid biopsy technique

$\mathrm{H}$ ISTORICALLY, the accuracy of CT-guided spinal biopsy has been reported to range between $61 \%$ and $100 \% .^{1,3-5,9,10,12,13,15,17,19,20}$ Two recent articles, including our own study, have demonstrated the poor diagnostic yield of percutaneous needle biopsy for vertebral osteomyelitis, with statistics as low as $19.0 \%$ positive microbiological cultures. ${ }^{8,18}$ However, in 2004, Lis et al. from Memorial Sloan Kettering Cancer Center evaluated 410 patients with known or suspected spinal malignancy and measured an $89 \%$ diagnostic yield per CT-guided biopsy. ${ }^{14}$ Our current study confirms the higher sensitiv- ity of image-guided percutaneous needle biopsy for vertebral neoplasms and analyzes which histology, imaging characteristics, and technical factors-including the use of a novel needle biopsy approach through a core biopsy track-provide the highest yield.

\section{Methods}

An institutional review board-approved retrospective chart review was performed for patients who underwent CT-guided vertebral bone biopsy during a 9-year period between January 2005 and October 2013. Each proce- 
dure was performed at University Hospitals Case Medical Center in Cleveland, Ohio, which serves as a Level II academic center.

A total of 213 percutaneous spine biopsies in 209 patients were collected through the retrospective Radiology Information Systems query. In our initial study analyzing the diagnostic yield of CT-guided percutaneous needle biopsy for vertebral osteomyelitis, these 213 vertebral bone biopsies were categorized according to the ordering clinician's suspicion and the indication listed on the radiology intervention requisition. Clinicians indicated a concern for infection in 84 requisitions and noninfectious etiology (the majority being neoplasms) in the remaining. We ascertained the low diagnostic yield of imageguided spinal biopsy for osteomyelitis, with only $19.0 \%$ of the cases showing positive results on microbiological cultures; these findings were published in Neurosurgical Focus in 2014. ${ }^{8}$

After our prior study, 9 additional vertebral bone biopsies were performed between October 2013 and May 2014; in total, 126 percutaneous vertebral biopsies were performed with the clinician's primary suspicion for neoplastic involvement. Those patients with a suspected lesion but without a clear clinical suspicion for neoplasm were not included in the current study. Furthermore, 4 of the 126 patients were excluded due to 1 case that involved a sacral biopsy and 3 patients with incomplete data within our electronic medical records. A total of 122 biopsies met the inclusion criteria. Among these, 1 patient underwent the procedure on 2 separate occasions; multiple months elapsed prior to the second biopsy, which was performed at a different vertebral level.

The ordering physician's suspicion for malignancy took into consideration cross-sectional imaging findings. Each of the 122 cases of spinal biopsy underwent a preprocedural CT scan; in the cases without a visible lesion on CT, an MRI or nuclear imaging study raised concern for a neoplastic process. This study did not focus on statistically comparing the efficacy of the preprocedural imaging.

Laboratory findings were obtained in accordance with the Society of Interventional Radiology guidelines for periprocedural management of coagulation status and hemostasis risk in moderate-risk percutaneous imagingguided interventions. ${ }^{16}$ Biopsy was performed in each case under CT guidance. A preprocedural 1- to 2-mm thinslice CT scan covering the area of interest was acquired, and the favorable biopsy approach was determined. The biopsy was performed with the patient under conscious sedation or, in selected cases of comorbidities or inability of the patient to lie prone, under general anesthesia. Local anesthesia was also used.

The biopsy approach varied depending on the lesion's location in the vertebral body or posterior elements. Figures 1-3 show examples of approaches used. A transpedicular approach was used in the majority of cases (68 of 122). A parapedicular approach was used in 7 cases and a transcostovertebral approach in 8 cases when a transpedicular approach was deemed less favorable. In 14 of 122 cases, a paraspinal soft-tissue component was identified and a paraspinal approach was used. In 25 cases, the lesion was located in the posterior elements (18 of 122) or in
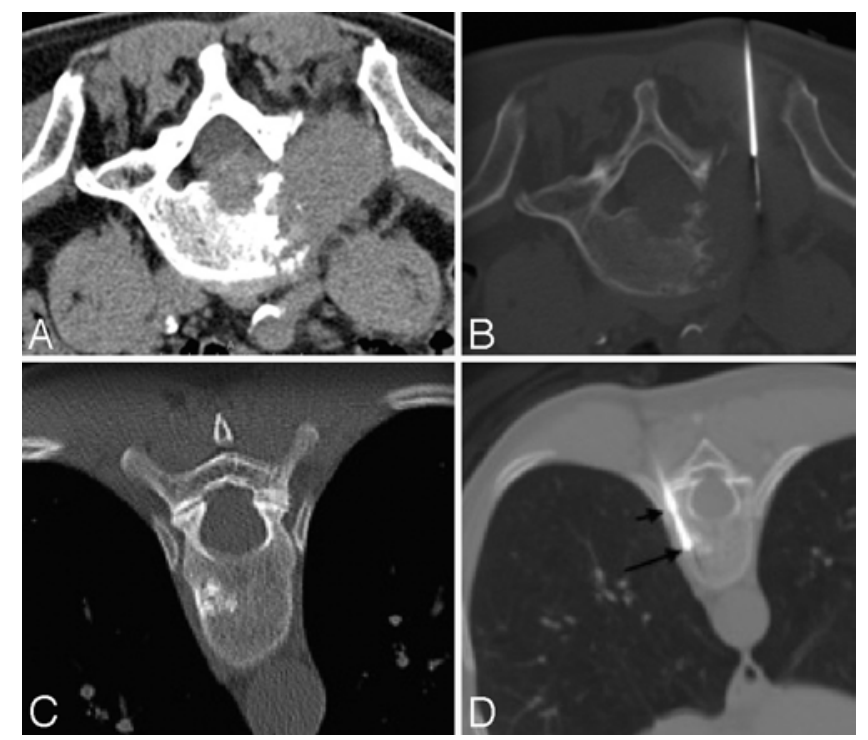

FIG. 1. A: A 65-year-old woman with a history of lung adenocarcinoma. A CT scan in a soft-tissue window demonstrates a lytic lesion at L-5 with a large adjacent soft-tissue component. B: Biopsy with a cutting needle within the soft-tissue component via a coaxial paravertebral technique. Pathological findings confirmed metastatic lung adenocarcinoma. C: A 55 -year-old woman with a history of breast cancer. A CT scan in a bone window demonstrates a sclerotic lesion within the T-10 vertebral body. D: Transcostovertebral coaxial approach with bone biopsy needle in place within the sclerotic lesion. A small black arrow marks the edge of the outer coaxial needle and the large black arrow indicates the tip of the inner trephine needle. Pathological findings confirmed metastatic breast adenocarcinoma.

the pedicles (7 of 122), and a corresponding approach was used targeting the respective lesion.

The biopsy technique varied depending on the lesion's imaging appearance as sclerotic, lytic, or mixed; the lesion's size; and the presence of a soft-tissue mass. In the latter case, this component was targeted preferentially, and a 14-gauge cutting biopsy needle (Temno; CareFusion) was used. In the presence of a destroyed or markedly thinned cortex and a lytic osseous lesion, a 14-gauge cutting biopsy needle was also used. In the presence of an intact cortex, a coaxial bone biopsy system was used and typically a bone core was obtained. However, in select cases (14 of 122) with an intact cortex and a lytic osseous lesion, the cortex was initially pierced with a coaxial bone biopsy system and subsequently a 14-gauge spring-loaded cutting biopsy needle was advanced into the lesion coaxially. This technique has been used since 2008 in efforts to minimize crush artifact from the larger introducer.

The biopsy system used was either a Laurane bone biopsy system (Laurane Medical) or a Bonopty bone biopsy system (Radi Medical Systems). The Laurane system consists of an 11-gauge external cannula with an internal stylet and a 12.5-gauge trephine biopsy needle. The Bonopty system consists of a 14-gauge external cannula and internal drill and a 16-gauge trephine biopsy needle. In both systems, the external cannula and stylet/drill were inserted into the bone, and then the internal stylet/drill was replaced by the trephine biopsy cutting needle. 

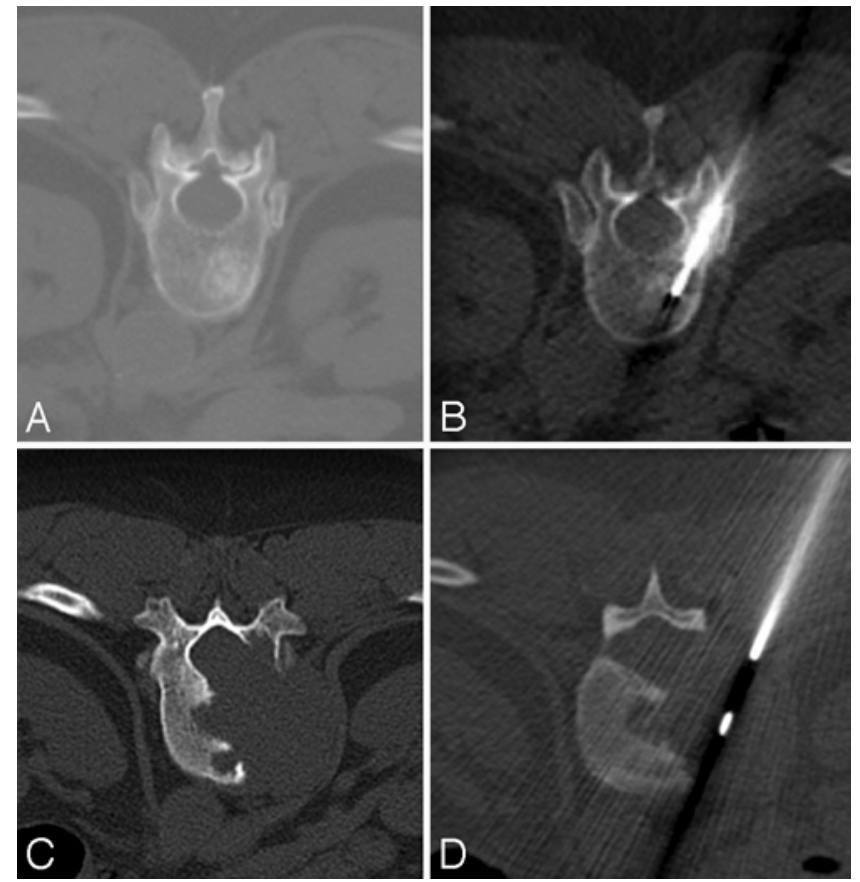

FIG. 2. A: A 45-year-old woman with a history of breast cancer. Axial CT demonstrates a sclerotic lesion within the T-12 vertebral body. B: Transpedicular biopsy approach through a coaxial bone biopsy system and the tip of the trephine needle within the sclerotic lesion. Pathological findings confirmed metastatic breast adenocarcinoma. C: A 35-yearold man without relevant medical history. Axial CT demonstrates a large lytic lesion with cortical destruction within the T-11 vertebral body. D: Parapedicular biopsy approach with a coaxial cutting needle through the lytic lesion. Pathological findings confirmed a giant cell tumor.

The number of core samples obtained varied on a caseby-case basis and depended on the size and location of the lesion biopsied. The radiologist used clinical judgment during each procedure to determine whether a single sample was sufficient or if additional biopsies should be performed.

\section{Results}

A total of $122 \mathrm{CT}$-guided percutaneous spinal biopsies were performed in which there was a strong clinical suspicion for neoplastic involvement. Of these 122 patients, 59 were male and 63 were female. The average age of the patient at the time of intervention was 58 years, with a range from 3 to 89 years of age. The majority of cases occurred at $>40$ years, with 36 patients between 50 and 59 years of age and 26 patients between 60 and 69 years old (charted in Fig. 4).

Each of the 122 patients who underwent the vertebral biopsy had a prior CT scan, of which $62(50.8 \%)$ demonstrated a lytic lesion, $28(23.0 \%)$ sclerotic, $16(13.1 \%)$ mixed sclerotic and lytic, 7 (5.7\%) soft-tissue masses, and $9(7.4 \%)$ without definitive visible lesion on CT. For these 9 cases without visualization on CT, other modalities, including MRI, identified the suspicious vertebral lesions.

The vertebral body was biopsied in 75 of the 122 cases $(61.5 \%)$. Thirty-five biopsies were of the posterior el-

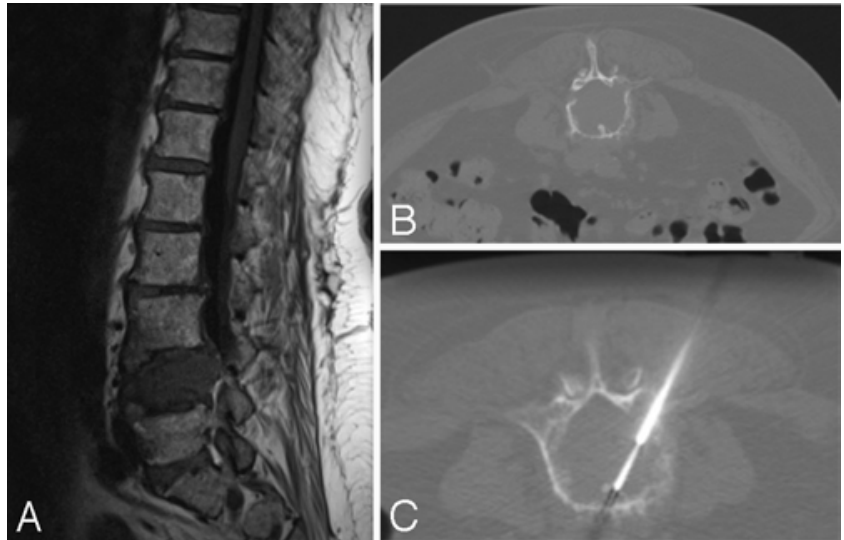

FIG. 3. A: A 64-year-old patient without relevant medical history. A sagittal T1-weighted image demonstrates a marrow-replacing lesion in the L-4 vertebral body. B: An axial CT image shows a lytic lesion in the vertebral body of $\mathrm{L}-4$. The cortex in the pedicles remains intact. C: $\mathrm{A}$ coaxial 11-gauge biopsy needle has been placed via a transpedicular approach; through the coaxial tract, a 14-gauge cutting needle was advanced into the lytic lesion. Pathological findings confirmed a plasmacytoma.

ements (28.7\%), and there were 12 cases of an adjacent paraspinal soft-tissue mass $(9.8 \%)$. The majority of biopsies were performed within the thoracolumbar spine (61 thoracic cases and 60 lumbar cases), with only 1 cervical vertebral biopsy. Sacral biopsies were not included in the cohort under investigation.

A core sample was obtained for each of the 122 biopsies $(100.0 \%)$, and fine needle aspiration (FNA) was obtained in 113 cases $(92.6 \%)$. Retrospective chart review of the 9 cases $(7.4 \%)$ without the addition of FNA did not specify why it was not performed. However, each radiologist used clinical judgment on a case-by-case basis to determine whether an FNA was needed or feasible; hence procurement of an FNA sample was forgone in these cases.

Histological findings in specimens obtained using FNA was nondiagnostic or unsatisfactory in 44 of the 113 cases $(38.9 \%)$, often with reports of hemorrhagic contents. Of the remaining 69 cases $(61.1 \%)$ in which the FNA was conclusive, metastatic malignant cells were reported in 44 (36.1\%) of the 122 total cases and were often of adenocarcinoma origin. Primary tumors were identified in 9 cases (7.4\%; 5 plasmacytomas, 2 blue cell tumors, and 2 blastic lesions). Nonspecific atypical cells were seen in 10 cases $(8.2 \%)$. The FNA clearly demonstrated no evidence for malignancy in the remaining 6 cases (4.9\%).

Histological findings in specimens obtained using core sampling provided more specific diagnoses. Of the 122 cases, only 6 were reported as nondiagnostic (4.9\%) and 12 cases were negative for disease $(9.8 \%)$. Core biopsy samples provided additional information in the remaining 104 cases $(85.3 \%)$. Metastases were identified in 62 cases $(50.8 \%) ; 24$ of these 62 cases were of breast origin $(38.7 \%)$ and 15 were lung metastases $(24.2 \%)$. A wide variety of metastases were seen in the remaining cases, but much less frequently; these are further detailed in Table 1 and Fig. 5.

Malignant primary bone lesions were seen in 15 of the 


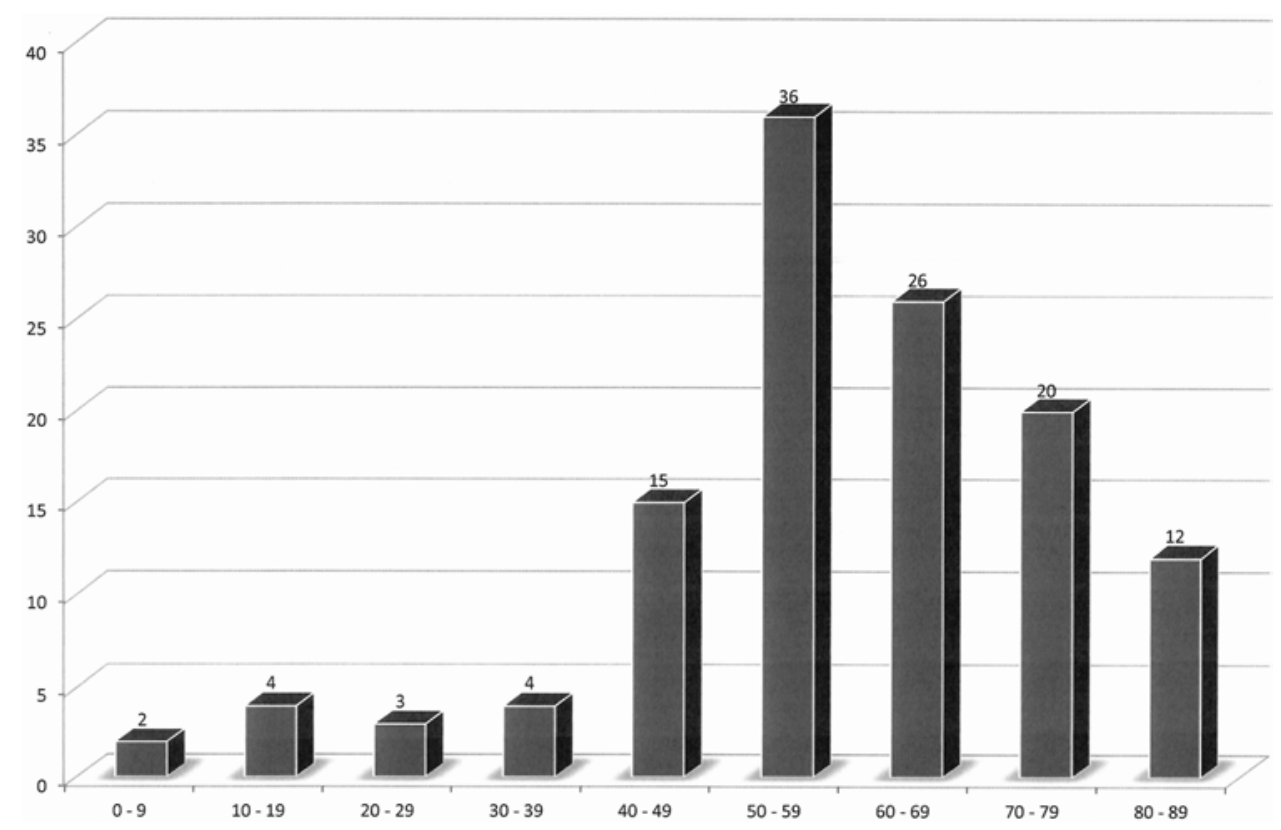

FIG. 4. Age demographic data of the patient cohort who underwent vertebral biopsy for neoplasm. Values on top of the bars are number of patients. $x$-axis $=$ years of age; $y$-axis = number of patients.

122 cases $(12.3 \%)$. Lymphoma and leukemia were identified in 5 cases $(4.1 \%)$, and 3 cases $(2.5 \%)$ of malignancies of unknown primary site (e.g., alveolar rhabdomyosarcoma of presumable testicular origin in a 16-year-old boy; high-grade, poorly differentiated epithelioid carcinoma in a 72-year-old man with subsequent palliative treatment; and atypical increased plasma cell and concern for plasma cell dyscrasia later diagnosed as monoclonal gammopathy of undetermined significance). Benign bone lesions, including benign primary bone tumors, benign atypical cells, neuronal tumors, and other pathologies, were seen in 19 cases (15.5\%). In regard to the 2 cases with benign atypical cells $(1.6 \%), 1$ case demonstrated a probable benign spindle cell lesion, which was later diagnosed as a benign myofibroma, and the second case demonstrated a benign hypocellular cartilaginous lesion. The details are outlined in Table 2.

In our last article analyzing the diagnostic yield of percutaneous needle biopsy for osteomyelitis, only $16 \mathrm{mi}$ crobiological samples of the 84 total biopsies identified a specific pathogen. Although the vast majority of cases were nondiagnostic, subsequent open biopsy was seldom performed; patients were typically treated with multiple weeks of intravenous antibiotics, without a clear answer for the initial presence of osteomyelitis. On the contrary, the question of metastatic or primary vertebral neoplastic involvement warrants follow-up, and the current study was able to determine the subsequent diagnosis of each vertebral lesion.

Ninety-four (77.0\%) core samples of the 122 total provided true-positive results, in which positive biopsy results were confirmed by either open biopsy or linear follow-up demonstrating progression of osseous metastatic disease. Fifteen (12.3\%) cases were deemed true negatives, with subsequent open biopsy, excision, or imaging follow-up (for at least 1 year) demonstrating benign results or no change on imaging. It is important to note that, in addition to the absence of a lesion, fractures, necrotic bone, Paget's disease, enostosis, and vascular lesions such as hemangiomas and hemangioblastomas were considered non-neoplastic lesions. Table 3 outlines each case of truenegative diagnosis.

A total of 13 cases $(10.7 \%)$ proved to be false negatives. The histology was negative or nondiagnostic and

TABLE 1. Percentage of metastases based on histological tissue

\begin{tabular}{lcr}
\hline Histological Etiology of Metastases & No. of Patients & $\%$ \\
\hline Breast & 24 & 38.71 \\
\hline Lung & 15 & 24.19 \\
\hline Renal & 4 & 6.45 \\
\hline Pancreatic & 3 & 4.84 \\
\hline Colon & 2 & 3.23 \\
\hline Bladder & 2 & 3.23 \\
\hline Prostate & 1 & 1.61 \\
\hline Melanoma & 1 & 1.61 \\
\hline Liver & 1 & 1.61 \\
\hline Esophageal & 1 & 1.61 \\
\hline Parotid & 1 & 1.61 \\
\hline Thyroid & 1 & 1.61 \\
\hline Scalp adenocarcinoma & 1 & 1.61 \\
\hline Maxillary adenoid cystic carcinoma & 1 & 1.61 \\
\hline Unknown & 4 & 6.45 \\
\hline Total & 62 & 100 \\
\hline
\end{tabular}




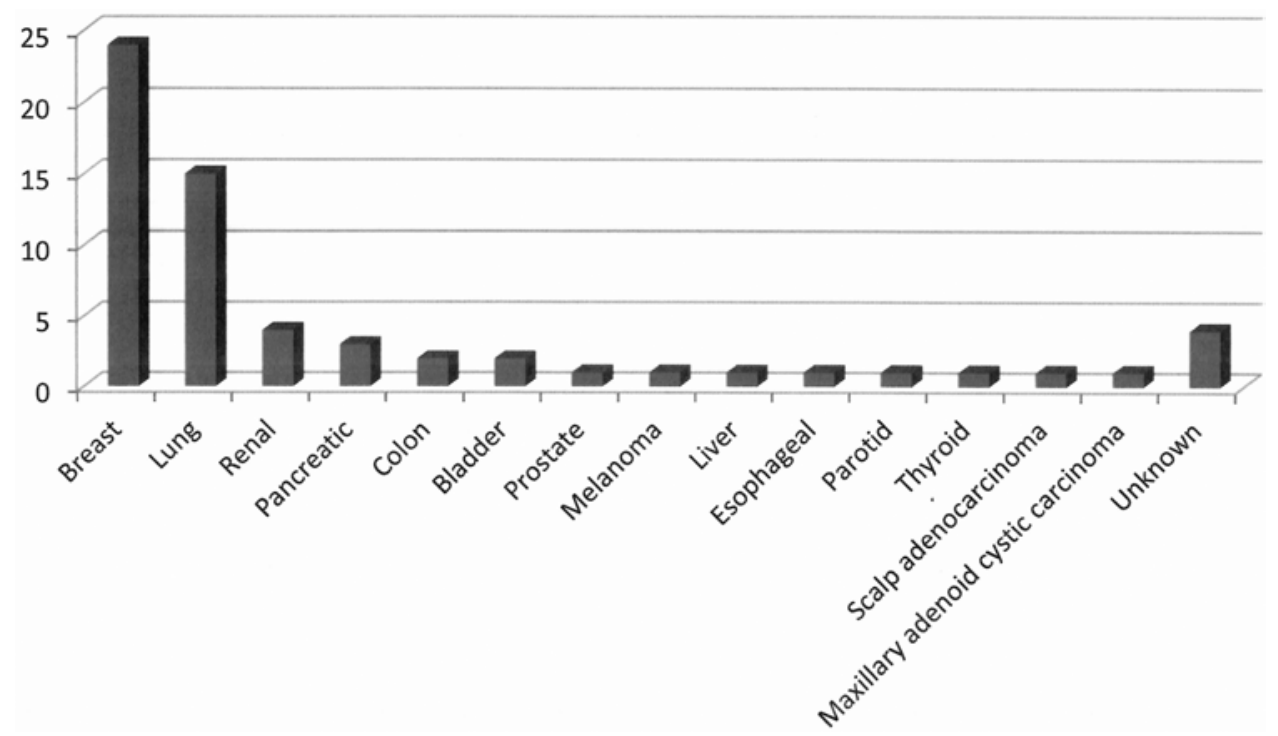

FIG. 5. Percentage of metastases based on histological tissue.

later deemed metastasis, primary tumor, or simply lost to follow-up (e.g., in the case of a suspicious paraspinal mass with nondiagnostic sample). Each case of a false-negative diagnosis is outlined in Table 4. There were no cases of false positives, i.e., there were 0 lesions that were deemed pathological and later determined to be benign. Therefore, the current study calculated the sensitivity of core biopsy as $87.9 \%$. Specificity and positive predictive value were both calculated as $100.0 \%$ due to the lack of false positives. The negative predictive value was calculated as $53.6 \%$.

Further analysis of the initial CT findings demonstrated lytic lesions in 62 cases $(50.8 \%)$, sclerotic lesions in 28 cases $(23.0 \%)$, mixed lesions in 16 cases $(13.1 \%)$, paravertebral soft-tissue masses in 7 cases $(5.7 \%)$, and no finding on CT in 9 cases $(7.4 \%$; lesions were identified by an alternative imaging modality). When comparing the imaging findings with the final diagnosis via follow-up, lytic lesions demonstrated 58 true positives of 62 and the highest sensitivity at $95.1 \%$. Sclerotic lesions established 15 true positives of 28 and sensitivity of $75.0 \%$; mixed sclerotic and lytic had 12 true positives of 16 and sensitivity of $80.0 \%$; soft-tissue mass showed 5 true positives of 7 and sensitivity of $83.3 \%$; and no lesion on CT resulted in 4 true positives of 9 and sensitivity of $80.0 \%$. It is important to highlight that, although comparable sensitivities were observed for sclerotic lesions and nonvisualized lesions, these categories demonstrated significantly fewer truepositive lesions. Specificity and positive predictive value were both calculated as $100.0 \%$ for all categories.

The technical approach did not appear to demonstrate any significant difference in diagnostic yield. Paraspinal

TABLE 2. Number and percentage of each histological finding

\begin{tabular}{|c|c|c|}
\hline Histological Finding & No. of Patients & $\%$ \\
\hline Nondiagnostic & 6 & 4.9 \\
\hline Negative & 12 & 9.8 \\
\hline Metastatic disease & 62 & 50.8 \\
\hline $\begin{array}{l}\text { Malignant primary bone tumor (multiple myeloma, } n=1 \text {; plasmacytoma, } n=10 \text {; Ewing's sarcoma, } \\
n=2 \text {; angiosarcoma, } n=1 ; M F H, n=1 \text { ) }\end{array}$ & 15 & 12.3 \\
\hline Lymphoma (B-cell, $n=4)$ and leukemia (acute myeloid, $n=1$ ) & 5 & 4.1 \\
\hline $\begin{array}{l}\text { Malignancy of unknown primary (metastatic alveolar rhabdomyosarcoma, } n=1 \text {; high-grade poorly } \\
\text { differentiated epithelioid carcinoma, } n=1 \text {; atypical plasma cell dyscrasia, } n=1 \text { ) }\end{array}$ & 3 & 2.5 \\
\hline $\begin{array}{l}\text { Benign primary bone tumor (Paget's disease, } n=3 ; G C T, n=3 \text {; osteochondroma, } n=1 \text {; osteoblas- } \\
\text { toma, } n=1 ; A B C, n=1 \text {; hemangioblastoma, } n=1 \text {; hemangioma, } n=1 \text {; bone island, } n=1 \text { ) }\end{array}$ & 12 & 9.8 \\
\hline Benign atypical cells (spindle cell lesion, $n=1$; benign hypocellular cartilaginous lesion, $n=1$ ) & 2 & 1.6 \\
\hline Neuronal (schwannoma, $n=1$; ganglioma, $n=1$ ) & 2 & 1.6 \\
\hline Other benign (fracture, $n=1$; necrotic bone, $n=1$; sclerotic bone with extensive remodeling, $n=1$ ) & 3 & 2.5 \\
\hline Total & 122 & 100 \\
\hline
\end{tabular}

$\mathrm{ABC}=$ aneurysmal bone cyst; $\mathrm{GCT}$ = germ cell tumor; $\mathrm{MFH}=$ malignant fibrous histiocytoma. 
TABLE 3. True-negative diagnoses and subsequent findings

\begin{tabular}{|c|c|c|}
\hline Demographic Data (age in yrs/sex/clinical Hx) & Imaging Result & True-Negative Diagnosis \\
\hline 73/M/prostate cancer & L-2 sclerosis & Paget's disease \\
\hline 88/F/metastatic breast cancer \& back pain & $\begin{array}{l}\text { No lesion per our imaging (but concern on outside } \\
\text { hospital report) }\end{array}$ & $\begin{array}{l}\text { Resolution of symptoms \& no change on } \\
\text { subsequent imaging exams }\end{array}$ \\
\hline 48/F/Hx of falls \& back pain & $\begin{array}{l}\text { Multilevel diffuse abnormal signal concerning for } \\
\text { marrow replacement }\end{array}$ & $\begin{array}{l}\text { Resolution of symptoms \& no change on } \\
\text { subsequent imaging exams }\end{array}$ \\
\hline 86/M/prostate cancer \& paralysis & $\begin{array}{l}\text { T-8 diffuse abnormal signal concerning for marrow } \\
\text { replacement }\end{array}$ & Healing fracture \\
\hline $\begin{array}{l}\text { 87/F/cervical cancer } \& \text { thoracic vertebral body } \\
\text { fracture }\end{array}$ & $\begin{array}{l}\text { Multilevel diffuse abnormal signal concerning for } \\
\text { marrow replacement }\end{array}$ & Healing fracture superimposed on anemia \\
\hline 49/M/incidental L-5 compression fracture & L-5 sclerosis concerning for compression fracture & Healing fracture \\
\hline 59/M/severe back pain & L-1 abnormal signal \& uptake on bone scan & Paget's disease \\
\hline 63/M/HIV, lymphoma, \& back pain & L1-2 abnormal signal \& uptake on PET & Necrosis \\
\hline 73/F/lt lower-extremity pain & L-2 dumbbell-shaped paraspinal tumor & Hemangioblastoma \\
\hline 69/M/prostate cancer \& fever & T9-10 abnormal signal changes & $\begin{array}{l}\text { No evidence of malignancy (treated as } \\
\text { disco-osteomyelitis) }\end{array}$ \\
\hline 53/F/back pain & L-5 sclerotic lesion & $\begin{array}{l}\text { No evidence of malignancy (no change in } \\
\text { appearance from prior yr) }\end{array}$ \\
\hline 56/M/prostate \& gastric adenocarcinoma & Multifocal sclerotic lesions w/ uptake on bone scan & $\begin{array}{l}\text { Sclerotic bone w/ active remodeling (sys- } \\
\text { temic treatment for gastric cancer) }\end{array}$ \\
\hline 73/M/prostate cancer \& back pain & T-6 abnormal signal \& uptake on bone scan & Paget's disease \\
\hline 58/F/osteosarcoma \& back pain & T7-10 rt paraspinal mass & Hemangioma \\
\hline 41/F/breast cancer & L-5 abnormal signal & Bone island \\
\hline
\end{tabular}

$\mathrm{Hx}=$ history.

and paravertebral approaches showed 12 true positives among a total of 14 cases and $85.7 \%$ sensitivity; transpedicular approach with 52 true positives among a total of 68 cases and $76.5 \%$ sensitivity; parapedicular approach with 6 true positives among 7 total cases and $85.7 \%$ sensitivity; transpedicular biopsy of the pedicle with 4 true positives among 7 total cases and $57.1 \%$ sensitivity; transcostovertebral approach with 6 true positives among 8 total cases and $75.0 \%$ sensitivity; and biopsy of the posterior elements with 14 true positives among a total of 18 cases and $77.8 \%$ sensitivity.

However, the combination of a bone biopsy needle and a cutting needle resulted in an optimal diagnostic yield. Bone biopsies, using a bone biopsy needle alone, provided 58 true positives among a total of 81 cases $(71.6 \%)$ with a sensitivity of $85.3 \%$, and cutting needles resulted in 22 true positives among a total of 27 cases (81.5\%) with a sensitivity of $88.0 \%$. Both a bone needle to penetrate the cortex and a cutting needle to obtain the core sample were used in 14 cases, with sufficient sample collected in each case and corresponding to 14 true positives with a sensitivity of $100.0 \%$. The small number of cases and lack of true negatives raise the question of whether this is statistically significant. A study with a larger sample size may be needed.

Limitations to this study must be acknowledged. Although each of the procedures was performed at the same institution by 3 board-certified musculoskeletal radiologists, varying intraprocedural circumstances prevented the standardization of sample acquisition. Each radiologist used clinical judgment during individual procedures to de- termine whether a single or additional core biopsy should be performed. If it appeared that sufficient tissue had been acquired from a core biopsy, FNA may have been avoided to prevent further distress to the patient. There was variability regarding sample type and size sent for microbiology analysis. The analysis of the data may have differed among the pathologists.

To collect the largest patient population for our study, the time frame of nearly 9 years meant that the period dated back to 2005 when the institution first began using a picture archiving and communication system. Patients who had undergone an image-guided percutaneous vertebral biopsy may have been unaccounted for, although we considered this to represent only a small number of cases, if in fact any cases were actually missed. Furthermore, the institution transitioned from paper documents to electronic medical records in the early 2000s. Data may have been lost during this transition. It is known that 3 cases in our current review had incomplete data in the electronic medical record and were omitted from the study.

\section{Discussion}

Cross-sectional imaging, including CT, MRI, bone scans, and PET/CT, has become routine in the initial staging and follow-up of cancers. Certain neoplasms demonstrate characteristic imaging findings, such as prostate cancer with sclerotic osseous metastases on CT and osteoblastic technetium-99m radiotracer uptake on bone scintigraphy. ${ }^{7}$ However, diagnosis by imaging is often confounded by the variety of presentations and multiple non- 
TABLE 4. False-negative diagnoses and subsequent findings

\begin{tabular}{ll}
\hline Initial False-Negative Diagnosis & \multicolumn{1}{c}{ Pathological Diagnosis } \\
\hline Nondiagnostic & Either metastatic breast or uterine endometrial cancer \\
\hline Nondiagnostic & Metastatic pulmonary non-small cell lung carcinoma \\
\hline Nondiagnostic & Metastatic gallbladder cancer \\
\hline Nondiagnostic & Multiple myeloma \\
\hline Nondiagnostic & Paraspinal mass, likely nerve sheath tumor \\
\hline Nondiagnostic & Extremely concerning lesions on imaging but lost to follow-up \\
\hline Negative & Metastatic breast cancer \\
\hline Negative & Metastatic prostate cancer \\
\hline Negative & Metastatic melanoma \\
\hline Negative & Malignant fibrous histiocytoma (per repeat percutaneous biopsy) \\
\hline Negative & Multiple myeloma \\
\hline Negative & Extremely concerning lesions on imaging but lost to follow-up \\
\hline Osteochondroma & Chondrosarcoma (Grade I; per excisional biopsy) \\
\hline
\end{tabular}

neoplastic etiologies with similar findings. A tissue biopsy is often needed for pathological confirmation.

Numerous publications have reported the high success rate of image-guided percutaneous vertebral biopsy, ranging from $61 \%$ to an ideal $100 \%$. $^{1,3-5,9,10,12,13,15,17,19,20}$ However, Sehn and Gilula reviewed 113 cases with suspected vertebral osteomyelitis and reported a meager $55.7 \%$ positive yield via histological and only $30.4 \%$ by microbiological investigation. ${ }^{18}$ We suspected a similar low yield from our patient population and performed a retrospective study, which demonstrated positive microbiological cultures in only 16 of $84(19.0 \%)$ samples. ${ }^{8}$ This discrepancy between our low diagnostic yield for vertebral osteomyelitis and frequently quoted higher statistics encouraged our own scrutiny of prior reports. We concluded that the previously proposed higher yield of vertebral osteomyelitis biopsy was either confounded by papers using inclusion criteria that were too narrow (which artificially elevated diagnostic yield) or by papers quoting statistics from other manuscripts that reported the general success of percutaneous spinal biopsy (without accounting for variable success between different pathologies).

Our next objective was to assess our success with percutaneous vertebral biopsy with the primary concern for neoplastic process and to confirm that our low yield for osteomyelitis was not secondary to a major flaw in technique. The current study collected 122 qualifying biopsies with a clinician's primary concern for neoplastic involvement. The overall sensitivity of CT-guided percutaneous spinal biopsy measured $87.9 \%$, substantially higher than our prior $19.0 \%$ microbiological yield for osteomyelitis. Hau et al. examined 359 patients with musculoskeletal lesions and acknowledged that the lowest success occurred with infectious diseases $(50.0 \%)$, significantly lower than neoplastic processes; furthermore, their article noted that spine biopsy had the lowest anatomical diagnostic accuracy $(61 \%)$, largely secondary to the low yield from infectious agents. ${ }^{11}$

In addition to confirming the high sensitivity of vertebral biopsy for neoplasm, our data pertaining to the neoplastic histological types procured match those of Lis et al. from Memorial Sloan Kettering Cancer Center. ${ }^{14}$ Metastatic disease was identified in 62 of 122 cases $(50.8 \%)$, with breast metastases the most common ( 24 cases, $38.7 \%$ ) and lung metastases the second most common (15 cases, $24.2 \%$ ), similar to Lis et al.'s reported $28 \%$ for breast and $7 \%$ for lung. Primary bone malignancies were second most common group of pathologies after metastases and were seen in 20 of the 122 vertebral cases (16.4\%), with 10 of the 20 cases diagnosed as plasmacytoma $(50.0 \%)$, compared with previously reported $6 \%$ primary bone tumors, with multiple myeloma/plasmacytoma attributed to $54.0 \%$. It is important to note that many variables account for the minute differences in percentages, including the initial clinical level of suspicion, demographic data, among others.

A comparison of the cross-sectional imaging findings with diagnostic yield confirmed the previously reported higher sensitivity for lytic lesions. Similar lower sensitivities for sclerotic lesions and lesions not visualized on CT have been described in the literature. For the former, although CT may have limitations in differentiating neoplastic from degenerative processes, MRI is particularly helpful in distinguishing between them. Pathological fractures typically demonstrate complete infiltration of the vertebral marrow, whereas compression fractures show less pronounced and more inhomogeneous signal changes with residual normal bone marrow; ${ }^{2}$ the lower sensitivity can therefore be attributed in part to difficulties in obtaining a sufficient sample and evaluating sclerotic bone in pathological investigation. Regarding the latter, lesions not visualized on CT but identified by MRI or PET imaging are more difficult to target and sampling error may play a role.

Our study did not quantitatively analyze the sensitivity for metastases with the presence of a known primary site. Biopsies were performed based on the initial indication listed on the CT and biopsy requisitions (e.g., history of malignancy) in combination with cross-sectional imaging findings. A few biopsies returned with unsatisfactory samples, which on subsequent imaging were determined to represent true lesions and therefore would underesti- 
mate the correlation between cancer history and vertebral metastases. Furthermore, our study did not retrospectively systematically rate the level of suspicion in each case. Biopsies were on occasion performed because of the concern for neoplasm or metastasis, although clinical suspicion was lower than typically needed to warrant the biopsy. In these cases, biopsy may have been necessary to rule out neoplastic involvement.

A general review of 108 suspected bone malignancies performed by Vieillard et al. demonstrated a diagnostic yield of $100 \%$ for metastatic disease compared with $83 \%$ for primary tumors and 58\% for hematological malignancies; this article also noted significantly less yield of vertebral lesions (66\%) compared with peripheral lesions $(85 \%){ }^{22}$ Another general review by Cronin et al. of new suspicious bone lesions in patients with a known primary carcinoma revealed the probability of only $2 \%$ for lesions other than metastatic disease. ${ }^{6}$ A cursory comparison of our own cases of known primary malignancy with suspicion for metastatic vertebral disease also suggested a high correlation. However, a few discordant results were present. There were 4 cases with history of malignancy and sclerotic lesion on CT but relatively benign etiologies (a single case each of degenerative changes, compression fracture, Paget's disease, and bone island). Three discordant lytic lesions were biopsied; 2 revealed plasmacytoma (with single histories of endometrial cancer and breast cancer) and 1 hemangioma (with initial history of osteosarcoma).

Fine needle aspiration obtains bits of tissue and cells by the mechanism of aspiration and requires a cytologist to analyze the results, whereas core needle biopsy extracts a larger sample through the use of a bigger needle. The larger sample acquired with the core needle has repeatedly proven higher diagnostic yield, as was demonstrated in our own study with diagnostic yield of $84.6 \%$ and sensitivity of $87.9 \%$ compared with the diagnostic yield of FNA (61.1\%). In addition to the increased risk of bleeding and tissue damage associated with the larger core needle, the larger introducer stylet of the core needle has been known to cause crushing artifact and insufficient/unsatisfactory sample size..$^{21,23}$

Despite the high yield obtained through the use of bone needles and cutting needles (yields of $71.6 \%$ and $81.5 \%$, respectively, in the current study and sensitivities of $85.3 \%$ and $88.0 \%$, respectively), this study evaluated techniques to minimize this crush artifact and optimize yield further. In 14 of the 62 lytic cases in which the osseous cortex was intact, the cortex was initially pierced with a coaxial bone biopsy system; subsequently, a 14-gauge spring-loaded cutting biopsy needle was advanced into the lesion coaxially. This technique provided sufficient sample in each of the 14 cases, with 14 true positives and a sensitivity of $100.0 \%$. It is important to note that the success with the small number of cases raises the question of whether this yield will hold with a larger sample size; further tests are needed to confirm the optimal yield for this cohort.

\section{Conclusions}

Our department's high yield of neoplastic tissue recov- ered from CT-guided percutaneous spinal biopsy supports prior reports and confirms the procedural utility, in contrast to our recent report of lower yield for vertebral osteomyelitis. ${ }^{8}$ Although there was no statistical difference in the biopsy approach (i.e., transpedicular, paraspinal, and so on), the use of a coaxial bone biopsy system to cut an intact osseous cortex prior to coaxial needle biopsy of lytic lesions minimized crush artifact and demonstrated $100.0 \%$ yield and sensitivity.

\section{References}

1. Babu NV, Titus VT, Chittaranjan S, Abraham G, Prem H, Korula RJ: Computed tomographically guided biopsy of the spine. Spine (Phila Pa 1976) 19:2436-2442, 1994

2. Baker LL, Goodman SB, Perkash I, Lane B, Enzmann DR: Benign versus pathologic compression fractures of vertebral bodies: assessment with conventional spin-echo, chemicalshift, and STIR MR imaging. Radiology 174:495-502, 1990

3. Bender CE, Berquist TH, Wold LE: Imaging-assisted percutaneous biopsy of the thoracic spine. Mayo Clin Proc 61:942-950, 1986

4. Brugières P, Gaston A, Voisin MC, Ricolfi F, Chakir N: CTguided percutaneous biopsy of the cervical spine: a series of 12 cases. Neuroradiology 34:358-360, 1992

5. Brugieres P, Revel MP, Dumas JL, Heran F, Voisin MC, Gaston A: CT-guided vertebral biopsy. A report of 89 cases. J Neuroradiol 18:351-359, 1991

6. Cronin CG, Cashell T, Mhuircheartaigh JN, Swords R, Murray M, O'Sullivan GJ, et al: Bone biopsy of new suspicious bone lesions in patients with primary carcinoma: prevalence and probability of an alternative diagnosis. AJR Am J Roentgenol 193:W407-W410, 2009

7. Davila D, Antoniou A, Chaudhry MA: Evaluation of osseous metastasis in bone scintigraphy. Semin Nucl Med 45:3-15, 2015

8. Garg V, Kosmas C, Young PC, Togaru UK, Robbin MR: Computed tomography-guided percutaneous biopsy for vertebral osteomyelitis: a department's experience. Neurosurg Focus 37(2):E10, 2014

9. Ghelman B, Lospinuso MF, Levine DB, O'Leary PF, Burke SW: Percutaneous computed-tomography-guided biopsy of the thoracic and lumbar spine. Spine (Phila Pa 1976) 16:736-739, 1991

10. Hao DJ, Sun HH, He BR, Liu TJ, Jiang YH, Zhao QP: Accuracy of CT-guided biopsies in 158 patients with thoracic spinal lesions. Acta Radiol 52:1015-1019, 2011

11. Hau A, Kim I, Kattapuram S, Hornicek FJ, Rosenberg AE, Gebhardt MC, et al: Accuracy of CT-guided biopsies in 359 patients with musculoskeletal lesions. Skeletal Radiol 31:349-353, 2002

12. Hua WB, Wu Q, Zhang B, Yang SH, Shao ZW, Xu WH, et al: Initial CT-guided percutaneous biopsy of vertebral lesions: Evaluation of its diagnostic accuracy and clinical value. J Huazhong Univ Sci Technolog Med Sci 35:569-573, 2015

13. Kornblum MB, Wesolowski DP, Fischgrund JS, Herkowitz $\mathrm{HN}$ : Computed tomography-guided biopsy of the spine. A review of 103 patients. Spine (Phila Pa 1976) 23:81-85, 1998

14. Lis E, Bilsky MH, Pisinski L, Boland P, Healey JH, O’Malley $\mathrm{B}$, et al: Percutaneous CT-guided biopsy of osseous lesion of the spine in patients with known or suspected malignancy. AJNR Am J Neuroradiol 25:1583-1588, 2004

15. Omarini LP, Garcia J: [CT-guided percutaneous puncturebiopsy of the spine. Review of 104 cases.] Schweiz Med Wochenschr 123:2191-2197, 1993 (Fr)

16. Patel IJ, Davidson JC, Nikolic B, Salazar GM, Schwartzberg MS, Walker TG, et al: Consensus guidelines for 
periprocedural management of coagulation status and hemostasis risk in percutaneous image-guided interventions.

J Vasc Interv Radiol 23:727-736, 2012

17. Poyanli O, Akan K, Unay K, Tangay C: CT-guided percutaneous transpedicular biopsy for the diagnosis of vertebral lesions. Acta Orthop Belg 74:503-506, 2008

18. Sehn JK, Gilula LA: Percutaneous needle biopsy in diagnosis and identification of causative organisms in cases of suspected vertebral osteomyelitis. Eur J Radiol 81:940-946, 2012

19. Stoker DJ, Kissin CM: Percutaneous vertebral biopsy: a review of 135 cases. Clin Radiol 36:569-577, 1985

20. Sucu HK, Ciçek C, Rezanko T, Bezircioğlu H, Erşahin Y, Tunakan M, et al: Percutaneous computed-tomographyguided biopsy of the spine: 229 procedures. Joint Bone Spine 73:532-537, 2006

21. Tehranzadeh J, Tao C, Browning CA: Percutaneous needle biopsy of the spine. Acta Radiol 48:860-868, 2007

22. Vieillard MH, Boutry N, Chastanet P, Duquesnoy B, Cotten A, Cortet B: Contribution of percutaneous biopsy to the definite diagnosis in patients with suspected bone tumor. Joint Bone Spine 72:53-60, 2005

23. Yaffe D, Greenberg G, Leitner J, Gipstein R, Shapiro M, Bachar GN: CT-guided percutaneous biopsy of thoracic and lumbar spine: A new coaxial technique. AJNR Am J Neuroradiol 24:2111-2113, 2003

\section{Disclosures}

The authors report no conflict of interest concerning the materials or methods used in this study or the findings specified in this paper.

\section{Author Contributions}

Conception and design: Garg, Kosmas. Acquisition of data: Garg, Josan. Analysis and interpretation of data: Garg, Josan. Drafting the article: Garg, Kosmas, Josan. Critically revising the article: Garg, Kosmas, Partovi, Bhojwani, Fergus. Reviewed submitted version of manuscript: Garg, Kosmas, Partovi, Bhojwani, Fergus, Young, Robbin. Approved the final version of the manuscript on behalf of all authors: Garg. Statistical analysis: Garg. Study supervision: Kosmas, Robbin.

\section{Correspondence}

Vasant Garg, Department of Radiology, University Hospitals Case Medical Center, Cleveland, OH 44106. email: vasant.garg@ gmail.com. 